\title{
Stellar population properties of the most massive globular clusters and ultra-compact dwarf galaxies of the Fornax cluster
}

\author{
Michael Hilker ${ }^{1}$ \\ ${ }^{1}$ European Southern Observatory, Karl-Schwarzschild-Str. 2, \\ D-85748, Garching bei München, Germany \\ email: mhilker@eso.org
}

\begin{abstract}
Most ultra-compact dwarf galaxies (UCDs) and very massive globular clusters reside in nearby galaxy clusters or around nearby giant galaxies. Due to their distance $(>4 \mathrm{Mpc})$ and compactness $\left(r_{\mathrm{eff}}<100 \mathrm{pc}\right)$ they are barely resolved, and thus it is difficult to obtain their internal properties. Here I present our most recent attempts to constrain the mass function, stellar content and dynamical state of UCDs in the Fornax cluster. Thanks to radial velocity membership assignment of $\sim 950$ globular clusters (GCs) and UCDs in the core of Fornax, the shape of their mass function is well constrained. It is consistent with the 'standard' Gaussian mass function of GCs. Our recent simulations on the disruption process of nucleated dwarf galaxies in cluster environments showed that $\sim 40 \%$ of the most massive UCDs should originate from nuclear star clusters. Some Fornax UCDs actually show evidence for this scenario, as revealed by extended low surface brightness disks around them and onsets of tidal tails. Multiband UV to optical imaging as well as low to medium resolution spectroscopy revealed that there exist UCDs with youngish ages, (sub-)solar $[\alpha / \mathrm{Fe}]$ abundances, and probably He-enriched populations.
\end{abstract}

Keywords. galaxies: star clusters, galaxies: abundances, galaxies: clusters: individual (Fornax)

\section{Introduction}

Most of the massive globular clusters (GCs) of our Milky Way show evidence of multiple stellar populations with differences in their light element abundances (see contributions of Piotto and Milone in this volume). A few GCs even exhibit spreads in iron abundance and probably age. Those are nuclear star cluster candidates whose host galaxies were disrupted during the assembly history of the Milky Way.

In galaxy clusters, disruption of low mass, nucleated galaxies was very common in the past. Indeed, in the Virgo and Fornax clusters there exists a large population of very massive and compact star cluster-like objects, called ultra-compact dwarf galaxies (UCDs). It was early speculated that they might be either "very bright GCs" or lower mass examples of "compact ellipticals like M32", or they might "represent the nuclei of disolved dE,Ns" (Hilker et al. 1999). In the recent years, more and more UCDs have been discovered, some very compact, some rather diffuse, filling up the previously unpopulated region between star clusters and dwarf galaxies in the luminosity-size plane (see figure 1). Different authors apply different definitions for UCDs, either invoking a lower luminosity/mass cut or a lower size cut. Since those definitions are not physically motivated, I consider as 'UCDs' all objects in the red box shown in figure 1. In general, UCDs are characterized by an upper envelope in the mass-size relation and enhanced dynamical mass-to-light ratios (Mieske et al. 2008b), roughly occurring at $>2 \times 10^{6} M_{\odot}$.

If one postulates that the complex GCs $\omega$ Centauri and M54 in the Milky Way and G1 


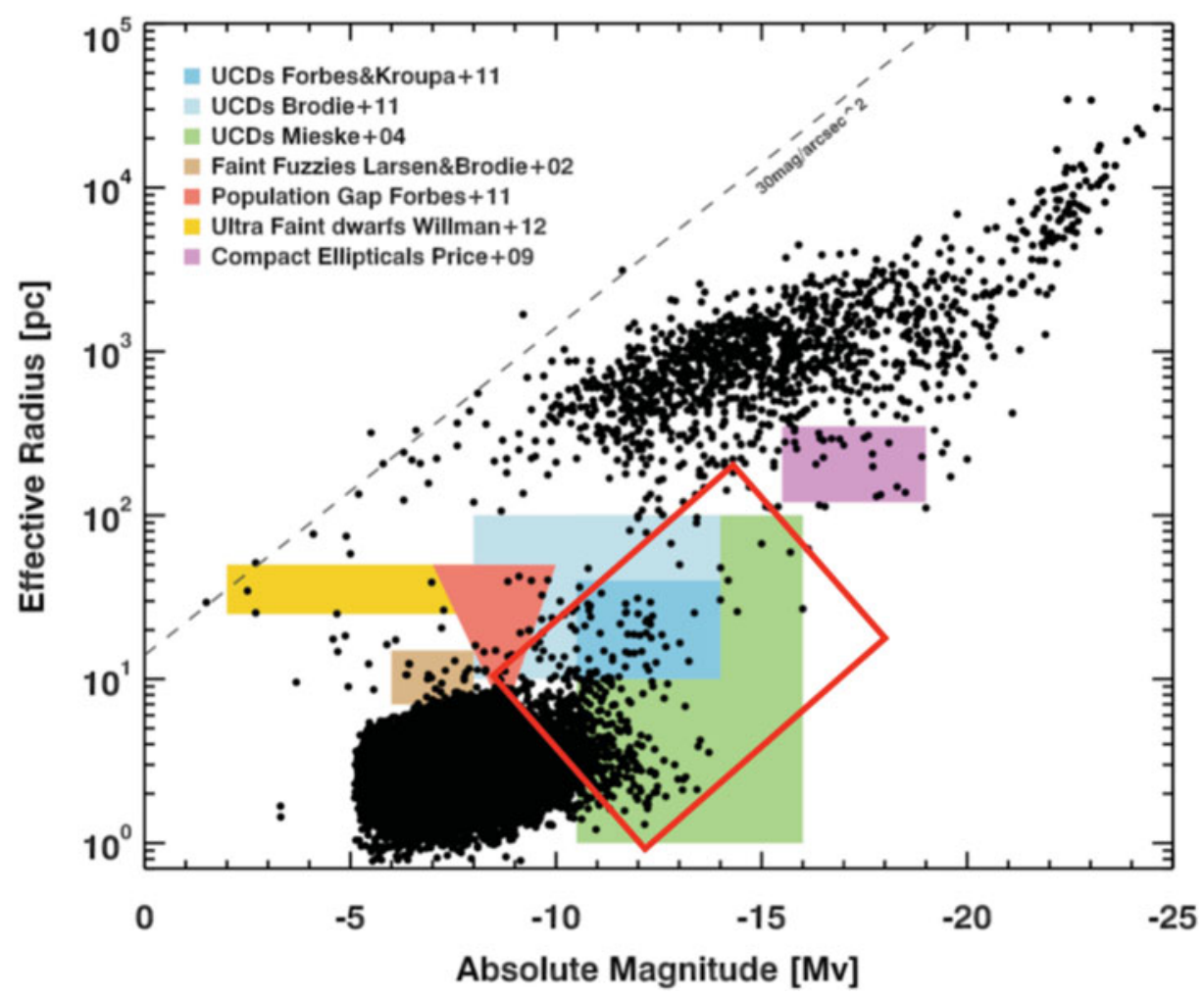

Figure 1. Luminosity-size plane of early-type stellar systems. The compilation is mainly based on similar samples shown in Misgeld \& Hilker (2011) and Norris et al. (2014). Coloured areas show different definitions of compact objects. Their references are given in the legend. The red rectangle encompasses the objects of interest for this contribution. The dashed line indicates the surface brightness limit of $30 \mathrm{mag} / \mathrm{arcsec}^{2}$.

in Andromeda are low-mass UCDs, one would expect that UCDs in general should also have complex star formation and chemical enrichment histories. However, due to the large distance (most are located beyond $4 \mathrm{Mpc}$ ) and thus unresolved nature of UCDs, multiple stellar populations in them are very difficult to detect and quantify. Most quantities have to be derived from images and spectra of their unresolved stellar populations. The determination of spatially resolved properties of UCDs requires space-based imaging and adaptive optics assisted spectroscopy.

\section{The most massive globular clusters in the Fornax cluster}

The Fornax cluster has a very well studied GC and UCD population. GC counts from photometric surveys revealed that there exist $\sim 6,450 \pm 700$ GCs within $83 \mathrm{kpc}$ projected distance around the central cluster galaxy NGC 1399 (Dirsch et al. 2003). Within $300 \mathrm{kpc}$ of NGC 1399 the GC number counts increase to $\sim 11,100 \pm 2,400 \mathrm{GCs}$ (Gregg et al. 2009, derived from the data of Bassino et al. 2006). The number of radial velocity confirmed GCs and UCDs around NGC1399 is 950 (Schuberth et al. 2010, Hilker, in prep.). Within $300 \mathrm{kpc}$ of NGC 1399 the sample of confirmed UCDs with masses above $5 \times 10^{6} M_{\odot}$ is rather complete and the photometrically selected GCs are well sampled below that limit. Thus, a mass function of UCDs and GCs can be constructed with high confidence (see also contribution of Schulz in this volume). The shape of that 


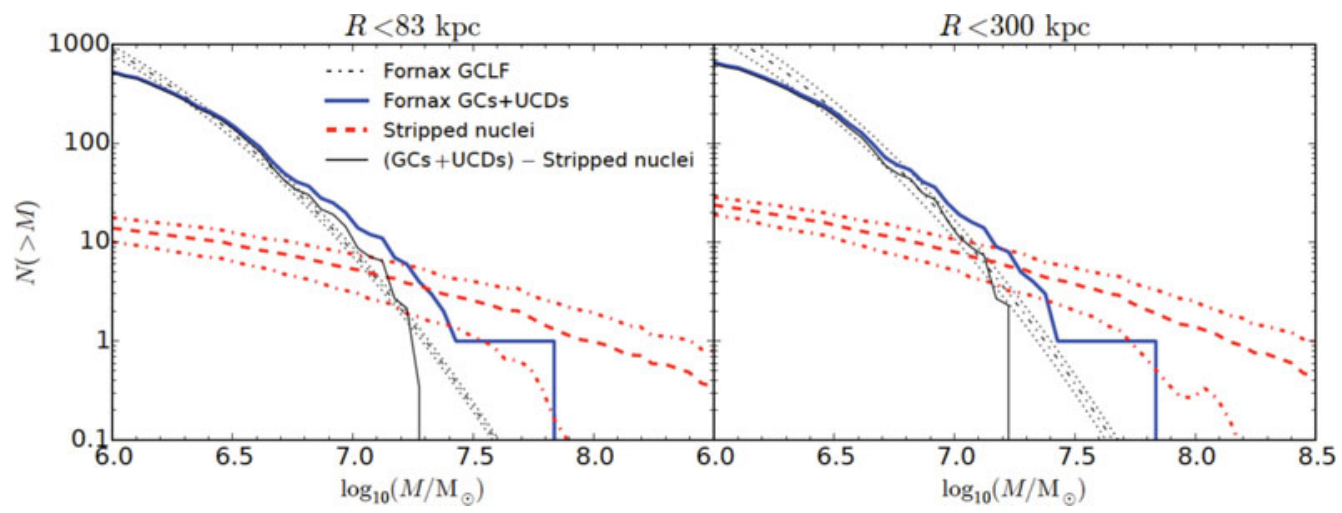

Figure 2. Cumulative mass function of simulated stripped nuclei in Fornax-like clusters within a projected distance of 83 (left panel) and $300 \mathrm{kpc}$ (right panel) from the central galaxy (dashed line with the standard deviation between clusters shown by dash-dotted lines) compared with GCs/UCDs in the Fornax cluster (thick solid line). The thin dash-dotted line shows the integrated Fornax GC luminosity function (GCLF) with the standard deviation given by dotted lines. The thin solid line shows the mean for the simulations subtracted from the Fornax GCLF. Figure taken from Pfeffer et al. (2015).

mass function was already presented in Hilker (2009), where we showed that the mass function above $3 \times 10^{5} M_{\odot}$ can be approximated with two power laws with slope exponents of -1.9 and -2.7 for objects below and above $2 \times 10^{6} M_{\odot}$, respectively. This shape is broadly consistent with the 'standard' (Gaussian) mass function of GCs. Thus, from this point of view, UCDs seem to be consistent with being drawn from the GC mass function (Mieske et al. 2012). However, the most massive UCD in Fornax, UCD3, exhibits a compact core and an extended stellar envelope of $\sim 90 \mathrm{pc}$ size (Evstigneeva et al. 2008), pointing rather to a remnant of a disrupted, nucleated dwarf galaxy.

We asked ourselves how many UCDs that originated from nuclei of disrupted galaxies are expected to contribute to the mass function in a Fornax cluster-like environment? We conducted cosmological simulations combined with a semi-analytic galaxy formation model and empirical prescriptions for the nucleation fraction and nuclei to galaxy mass fraction to identify stripped nuclei of disrupted satellite galaxies (Pfeffer et al. 2014). In figure 2 we show the cumulative mass function of the simulated stripped nuclei and compare it with the mass function of observed GCs/UCDs with masses $>10^{6} M_{\odot}$. As one can see, stripped nuclei can only explain the number of very massive UCDs. For masses larger than $10^{7} M_{\odot}$ we predict stripped nuclei account for $\sim 40 \%$ of GCs/UCDs. For masses between $10^{6}$ and $10^{7} M_{\odot}$ the stripped nuclei account for only $\sim 2.5 \%$ of GCs/UCDs. Thus, the majority of lower mass UCDs should be of star cluster origin, either formed as very massive genuine globular cluster or being the result of merged super star cluster complexes (e.g., Fellhauer \& Kroupa 2002, Brüns et al. 2009).

Indeed, there exist young massive star clusters that occupy the same space in the mass-size plane as UCDs (Kissler-Patig et al. 2006). Thus, mass and size alone are not indicative of the origin of UCDs.

\section{Internal properties of ultra-compact dwarf galaxies}

The nature of UCDs can be revealed, or at least constrained, by analysing the spatially resolved surface brightness profiles and internal kinematics and by studying the properties of their unresolved stellar population. A supermassive black hole (SMBH) and/or and 

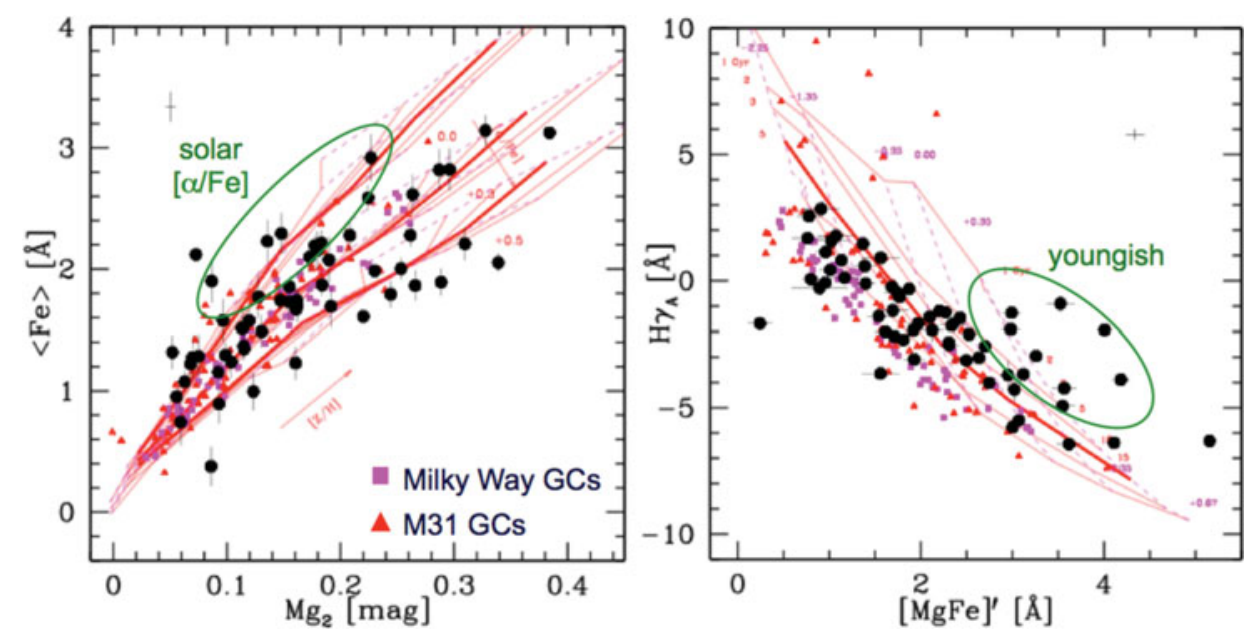

Figure 3. Lick index measurements of $\sim 50$ UCDs and massive GCs in the core of the Fornax cluster (black dots) compared to Milky Way and M31 GCs (as indicated). Left panel: $\mathrm{Mg}_{2}$ versus iron index, which allows to differentiate $[\alpha / \mathrm{Fe}]$ abundances. Right panel: Metallicity index $[\mathrm{MgFe}]$ ' versus the Balmer index $\mathrm{H}_{\gamma_{A}}$, which breaks the age-metallicity degeneracy. The model grids are based on SSP models from Thomas et al. (2003). Objects with (sub-)solar $[\alpha / \mathrm{Fe}]$ values and young ages are encircled by green ellipses.

extended star formation history would favour their origin as stripped nuclei of a dwarf galaxy. A high metallicity, i.e. not obeying the mass-metallicity relation of nuclei, would rather point to a star cluster origin in an enriched (maybe merger-induced) environment.

Evstigneeva et al. (2008) revealed positive colour gradients in the resolved light profiles of some UCDs in Fornax and Virgo. A blue nucleus and a red envelope are consistent with the colour differences of nuclei and stellar body in nucleated dwarf ellipticals (Lotz et al. 2004), where nuclei are formed from merging, metal-poor GCs.

In a recent study of the structural composition and clustering properties of 97 UCDs in the halo of NGC 1399, we found evidence for faint stellar envelopes around several UCDs with effective radii of up to $90 \mathrm{pc}$ (Voggel et al. 2015). One particularly extended UCD shows clear signs of tidal tails extending out to $\sim 350 \mathrm{pc}$. We also detected, in a statistical sense, a local overdensity of blue GCs on scales of $\leqslant 1 \mathrm{kpc}$ around UCDs. These could either be remnant GCs of a formerly rich GC system around a disrupted dwarf galaxy, or surviving star clusters of a merged super star cluster complex (e.g., Brüns et al. 2009).

Combining GALEX NUV/FUV with optical photometry, Mieske et al. (2008) showed that the location of several Fornax UCDs and massive GCs in the (FUV-NUV) versus (NUV-V) plane can only be explained with He-enhanced SSP models. This might be a hint to multiple populations in these GCs/UCDs, since He enhancement is also observed in the second generation stars of multi-population Galactic GCs.

Deep VLT/FORS2 spectroscopy on $\sim 50$ bright GCs $\left(M_{V}<-9 \mathrm{mag}\right)$ allowed us to measure Lick indices (Hilker, Puzia, et al., in prep.). In Fig. 3 we show two planes of Lick indices that constrain the $[\alpha / \mathrm{Fe}]$ abundances and break the age-metallicity degeneracy, respectively. Besides old GCs with enhanced $[\alpha / \mathrm{Fe}]$ abundances, there also exist objects with (sub-solar) $[\alpha / \mathrm{Fe}]$ values and some metal-rich intermediate-age GCs/UCDs (2-7 Gyr). Those might have had their origin in dwarf galaxies with low star formation rates and/or as nuclear star clusters with recurrent star formation episodes.

The most convincing case of a stripped nuclei origin of a UCD is the discovery of a $\mathrm{SMBH}$ in M60-UCD1, one of the most massive and densest UCDs in the Virgo cluster 

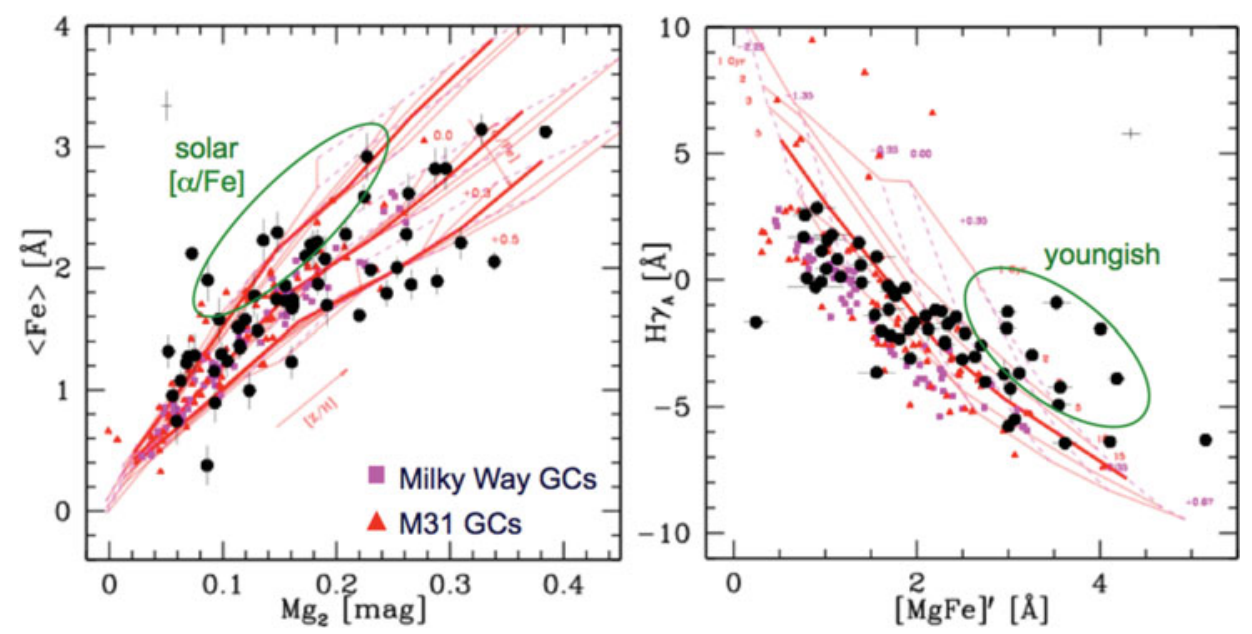

Figure 4. Predicted masses of central black holes in stripped nuclei as function of stripped nucleus mass. The mean and 1- $\sigma$ confidence interval for the simulations (grey dots) are given by the solid and dashed lines, respectively, using bin sizes of 100 objects. The typical $1-\sigma$ confidence interval in $\mathrm{MBH}$ is 0.5 dex. For comparison, we also show the black hole mass of M60-UCD1 (Seth et al. 2014), the inferred black hole masses of UCDs assuming elevated mass-to-light ratios are due to central black holes (Mieske et al. 2013) and the limits for central black holes in the GCs $\omega$ Cen and G1 (Lützgendorf et al. 2013). For the observed UCDs and GCs, objects with implied black hole masses of zero are given by triangles at the bottom of the figure. Figure taken from Pfeffer et al. (2015).

(Seth et al. 2014). The SMBH comprises $15 \%$ of the UCD's total mass. In our simulations of stripped nuclei (Pfeffer et al. 2015) we predict the masses of their central black holes (BHs) as function of stripped nuclei mass (see figure 4). The SMBH mass of M60-UCD1 falls on top of the simulations, and, interestingly, also the inferred BH masses of UCDs with elevated mass-to-light ratios (Mieske et al. 2013) are very much consistent with the simulations. More AO-assisted spectroscopic observations are underway to prove or disprove the SMBH hypothesis for UCDs with high dynamical mass-to-light ratios.

\section{Summary and outlook}

Our general conclusions from the findings on UCDs and massive GCs in the Fornax cluster presented in this contribution can be summarized as follows:

- UCDs are defined through an upper envelope in the mass-size relation and enhanced dynamical mass-to-light ratios - roughly occurring at $>2 \times 10^{6} M_{\odot}$.

- UCDs share properties of nuclei as well as young massive star clusters, e.g. the mass-size relation. They are a mixed bag of objects.

- The mass function of massive GCs/UCDs in Fornax shows a break at $\sim 2 \times 10^{6} M_{\odot}$. There is no excess of UCD-mass objects. Thus, they are mostly of 'star cluster origin'.

- Stripping of nucleated galaxies in a cosmological framework cannot explain the total number of UCDs, but $\sim 40 \%$ of UCDs with a mass $>10^{7} M_{\odot}$ are compatible with stripped nuclei.

- M60-UCD1 has a SMBH, which makes up $15 \%$ of the UCD's mass. This is direct evidence for the stripping scenario as valid channel to form UCDs.

- Extended stellar envelopes and overdensities of star clusters around UCDs might hint at the accretion of nucleated dwarf galaxies or at a dissolving super star clusters that were formed from merged star cluster complexes. 
Distinguishing stripped nuclei from super star clusters or massive GCs is a difficult task. Many internal properties of UCDs can be explained by both scenarios. More measurements of SMBHs in UCDs, derivation of their star formation histories and light element abundances are needed to unveil the nature of individual UCDs, and thus build up statistically meaningful samples for UCDs of different flavours.

\section{References}

Bassino, L. P., Faifer, F. R., Forte, J. C., Dirsch, B., Richtler, T., Geisler, D., \& Schuberth, Y. 2006, $A \mathscr{E} A, 451,789$

Brüns, R. C., Kroupa, P., \& Fellhauer, M. 2009, ApJ, 702, 1268

Brodie, J. P., Romanowsky, A. J., Strader, J., \& Forbes, D. A. 2011, AJ, 142, 199

Dirsch, B., Richtler, T., Geisler, D., Forte, J. C., Bassino, L. P., \& Gieren, W. P. 2003, AJ, 125, 1908

Evstigneeva, E. A., Drinkwater, M. J., Peng, C. Y., Hilker, M., De Propris, R., et al. 2008 AJ, 136,461

Fellhauer, M. \& Kroupa, P. 2002, MNRAS, 330, 642

Forbes, D. A., \& Kroupa, P. 2011 PASA, 28, 77

Forbes, D. A., Pota, V., Usher, C., Starder, J., Romanowsky, A. J., et al. 2013 MNRAS, 435, L6

Gregg, M. D., Drinkwater, M. J., Evstigneeva, E., Jurek, R., Karick, A. M., Phillipps, S., Bridges, T., Jones, J. B., Bekki, K., \& Couch, W. J. 2009 AJ, 137, 498

Hilker, M., Infante, L., Vieira, G., Kissler-Patig, M., \& Richtler, T. 1999, A\&AS, 134, 75

Hilker, M. 2009, in: S. Roeser (ed.), Reviews in Modern Astronomy: Formation and Evolution of Cosmic Structures, Vol.21, (Wiley-VCH Verlag GmbH \& Co. KGaA), p. 199

Kissler-Patig, M., Jordán, A., \& Bastian, N. 2006 A\&A, 448, 1031

Larsen, S. S., \& Brodie, J. P. 2002 AJ, 123, 1488

Lotz, J. M., Miller, B. W., \& Ferguson, H. C. ApJ, 613, 262

Lützgendorf, N., Kissler-Patig, M., Neumayer, N., Baumgardt, H., Noyola, E., et al. 2013 A\&A, 555,26

Mieske, S., Hilker,M., \& Infante, L. 2004 A\&A A, 418, 445

Mieske, S., Hilker,M., Bomans, D. J., Rey, S.-C., Kim, S., Yoon, S.-J., \& Chung, C. 2008 A\&A, 489,1023

Mieske, S., Hilker,M., Jordán, A., Infante, L., Kissler-Patig, M., et al. 2008 A\& A, 487, 921

Mieske, S., Hilker, M., \& Misgeld, I. 2012 A $\mathscr{E} A, 537,3$

Mieske, S., Frank, M. J., Baumgardt, H., Lützgendorf, N., Neumayer, N., \& Hilker, M. 2013 $A \& A, 558,14$

Misgeld, I. \& Hilker, M. 2011, MNRAS, 414, 3699

Norris, M. A., Kannappan, S. J., Forbes, D. A.; Romanowsky, A. J., Brodie, J. P., et al. 2014 MNRAS, 443, 1151

Pfeffer, J., Griffen, B. F., Baumgardt, H., \& Hilker, M. 2014, MNRAS, 444, 3670

Pfeffer, J., Hilker, M., \& Baumgardt, H. 2015 MNRAS, submitted

Schuberth, Y., Richtler, T., Hilker, M., Dirsch, B., Bassino, L. P., Romanowsky, A. J., \& Infante, L. $2010 A \& 3 A, 513,52$

Seth, A. C., van den Bosch, R., Mieske, S., Baumgardt, H., \& Brok, M. Den, et al. 2014, Nature, 513,398

Thomas, D., Maraston, C. \& Bender, R. 2003 MNRAS, 339, 897

Voggel, K., Hilker, M., \& Richtler, T. 2015 A $\& A$, accepted

Willman, B., \& Strader, J. 2012 AJ, 144, 76 